\title{
Consciousness and brain
}

\begin{abstract}
After rapid development of science and technology, consciousness has been the subject of intense research in psychology, neuroscience and quantum mechanics. Neuroscience is the most promising field and has made significant progress in understanding the structure and function of brain. Human brain has 100 billion neurons and 100 trillion constantly changing connections. At the root of all our thoughts, emotions and behaviors is the communication between neurons within our brains. Brain waves are produced by synchronized oscillations of group of neurons communicating with each other in very specific frequencies. Hence, Brain wave is rhythmic neural activity in the brain. Brain waves are classified in five bands - delta, theta, alpha, beta, gamma - with different frequencies. The article clearly explains and concludes that consciousness is a function of the brain wave frequencies in these bands.
\end{abstract}

Keywords: consciousness, neuron, brain wave, consciousness constant, fourier transform
Volume I Issue 4 - 2018

\author{
Tapan Das \\ Professional Engineers Ontario, Canada
}

Correspondence: Tapan Das, Professional Engineers Ontario, Ottawa, Canada, Tel 61342245 4I, Email tkdas@rogers.com

Received: July 17, 2018 | Published: August 31, 2018

\section{Introduction}

Consciousness has been a mystery from very early civilization. Every religion and many philosophers have tried to explain consciousness. Dualism believes that consciousness is different from the body and has no relation to the brain. Monism believes that consciousness is part of brain activity and brain acts like supercomputer which can do all necessary functions including consciousness. Three recent dominant theories are quantum consciousness, consciousness as universal property (panpsychism), and cognitive theory. But, nothing has been proven yet. Author has taken a totally different view that consciousness is the product of brain wave frequencies and consciousness constant C. Relation of brain wave frequencies with consciousness have been experimentally proven by neuroscientists.

\section{Brain waves and consciousness}

Consciousness is created by brain waves. When our brain is dead, we are dead, and we have no consciousness. Average human brain weighs around 2.5 pounds and has 100 billion brain cells called neurons and three trillion connections between the neurons. Neurons communicate with each other through axon and dendrites. A neuron has several dendrites but one axon that may be as long as two feet. Dendrites receive signals from other neurons and axons transmit signal to other neurons.

At the root of all our thoughts, behaviors and emotions is the communication between neurons in our brains. ${ }^{1,2}$ Brainwaves are produced by synchronized electrical pulses from masses of neurons communicating with each other. Brain wave is rhythmic or repetitive neural activity in the brain. This interaction between the neurons giving rise to brain wave at a certain frequency is different from the firing frequency of individual neurons. The major brain wave frequencies are - Delta, Theta, Alpha, Beta, and Gamma:

I. Delta: frequency range is $0.5-4$ cycles per second $(\mathrm{Hz})$. They are generated in dreamless and deepest meditation.

II. Theta: frequency range is $4-8 \mathrm{~Hz}$. They are generated in relaxed state.

III. Alpha: frequency range is $8-12 \mathrm{~Hz}$. They are generated in mental coordination, calmness and alertness.
IV. Beta: frequency range is $12-40 \mathrm{~Hz}$. They are generated in focused mental activity, problem solving, judgment and decision making.

V. Gamma: frequency range is $40-100 \mathrm{~Hz}$. They are generated in cognitive functioning, learning and information processing.

As we see that the frequency of the brain wave changes with the mental activity. Hence, mental activity is a function of brain wave frequency. When the brain wave frequency is high, brain is very active. When we are in the state of deep sleep or meditation, the brain activity is very low and brain wave frequency is $4 \mathrm{~Hz}$ and less. When we are in deeply relaxed state, then the brain activity is low and the brain wave frequency is between $4 \mathrm{~Hz}$ to $8 \mathrm{~Hz}$. When we are calm and alert, our brain activity is higher and the brain wave frequency is between 8 and $12 \mathrm{~Hz}$. When we are engaged in mental activities like problem solving, decision making etc., our brain activity is even higher and brain wave frequency is between $12 \mathrm{~Hz}$ and $40 \mathrm{~Hz}$. When we are engaged in extremely busy mental function, our brain activity is very high and the brain wave frequency is between $40 \mathrm{~Hz}$ and $100 \mathrm{~Hz}$. Hence, it is obvious that brain activity is proportional to the brain wave frequency (BWF). Brain activity needs brain energy. Hence, brain energy is proportional to the brain wave frequency. Consciousness is related to brain activity, hence brain energy. Hence, consciousness is proportional to the brain wave frequency. If physical constant of consciousness is Consciere $\mathrm{C}$, and brain wave frequency is $f$, then consciousness is $=$ C.f.

Now the immediate question will be where does constant Consciere C come from? Newton explained gravity by the theory that every mass has gravitational force and introduced a gravitational constant G. Hence, gravity is the product of the mass and gravitational constant G. Similarly, light energy increases with frequency i.e. blue light has more energy than red light. This is because; light consists of light particles called photons. Planck explained the energy of a photon by introducing a Planck constant $h$. Hence, photon energy is the product of the frequency of photon $f$ and Planck constant $h$ i.e. h.f. The energy of gas goes up with temperature, because the energy of a particle is the product of Boltzmann constant $\mathrm{k}_{\mathrm{B}}$ and temperature T i.e. $\mathrm{k}_{\mathrm{B}} \mathrm{T}$. There are physical constants in science. Similarly, Consciere $C$ is a physical constant for consciousness. It only applies to brain waves. In any mental activity, the dominant waves will be in the ranges related to the type of activity. For example, when we are engaged in problem 
solving our dominant brain waves will be in Beta range, but there will be some brain waves from Alpha, Theta, and Gamma ranges.

Now the next question will be, "what is the value of Consciere C?" Brain energy is extremely low to measure with current tools. Once it is possible to measure brain energy, we can calculate the value of Consciere $\mathrm{C}$, by using a mathematical tool called Fourier transform (Appendix 1).

Patients are made unconscious by anaesthesia for surgical operation. If unconscious means that the patient has no consciousness, then the term is wrong. The patient has still consciousness. The patient has still heart beat and is breathing. Heart beat and breathing are controlled by lower part of brain called brainstem. Across the whole brain, the brain wave has a low frequency around $1 \mathrm{~Hz}$ which is in low Delta range. ${ }^{3}$ Within localized brain regions neurons are active for a few hundred milliseconds, then shut off for a few hundred milliseconds. When this area was active, it is unlikely that the other areas were not active. Hence could not send information to other areas. This flickering of activity creates the low brain wave frequency. So, instead of using the word 'unconscious', we should use the word 'unresponsive'.

Anaesthesia is given to a patient so that she does not feel any pain due to surgery. Pain starts at the source of an injury or inflammation. The spinal cord carries the pain message from its receptors all the way up to the brain, where it is received by the thalamus and sent to the cerebral cortex. ${ }^{4}$ Cerebrum is the largest part of the brain the part of the brain that processes the message. It has two hemispheres, left and right. The left hemisphere receives most inputs from and controls mostly the right side of the body. The right hemisphere deals mostly with the left side of the body. Thalamus is the gateway to the cortex and works as a command center. Any single individual area of the brain cannot be identified as "pain center". Pain is a combination of sensory, affective and cognitive components that requires multistage processing including first-order processing in subcortical structures and higher-order processing in the cerebral cortex. Each stage and component is mediated not by one, but by a multitudinous group of regions. Anaesthesia interrupts normal firing patterns of neurons and the ability of different brain regions to communicate with each other, thus nullifying the creation of pain.

There are many cases of near death experiences (NDEs) with patients, who were according to all known laws of physics, technically in a state of coma due to heart attack. The patients, after being brought back to awareness, explained in detail what was happening around while the patient was technically in coma. One of the most famous proof is the story of Maria, a migrant worker who had an NDE during a cardiac arrest at a hospital in Seattle in $1977 .{ }^{5}$ She later told her social worker that while doctors were resuscitating her, she found herself floating outside the hospital building and saw a tennis shoe on a thirdfloor window ledge, which she described in some detail. The social worker went to the window Maria had indicated, and not only found the shoe but said that the way it was placed meant there was no way Maria could have seen all the details she described from inside her hospital room. As mentioned before, in coma, the neurons in localized brain areas are active cyclically for few hundred milliseconds. Is it possible that periodic activity of localized patch of neurons gives rise to NDE? It needs lot of research work by neuroscientists.

A study at the University of Michigan, published in 2013, describes that George Mashour, an anesthesiologist from the University of Michigan and his colleagues ${ }^{6}$ took anesthetized rats and stopped their hearts. Within 30 seconds, the rats' EEG brain signals flatlined - but first they spiked, with an intensity that suggested that different parts of the brain were communicating with one another even more actively than when the rats were awake. If we could establish that spikes in neural activity occur in a dying human brain like the ones seen in rats, that could both help explain near-death experiences and give us some clues about the neurobiological nature of consciousness. Due to obvious ethical reasons, it is not possible to carry out similar experiments on humans. This study on rats at least illuminates the possibility that to explain near-death experiences we need to consider the connection between the brain and consciousness.

Consciousness has been an intriguing and puzzling subject from very early civilization. It has been analyzed from subjective and objective aspects, monism and dualism aspects, classical physics and quantum mechanism aspects, religious aspect, and philosophical aspect. But still it remains a mystery. The best and only way out of it is to accept that consciousness must be physical property of brain waves. The logic is:

1. Science has proven that brain waves exist.

2. A person who is brain dead is not conscious and brain waves do not exist.

3. The nature and state of consciousness depend on the frequency of brain waves.

4. Term 'unconscious' should be replaced by 'unresponsive' because we are always conscious until we are dead i.e. our brain is dead hence no consciousness.

\section{Conclusion}

Consciousness is a fundamental property of brain waves. Newton introduced gravitational constant as fundamental property of mass to solve gravity. Planck introduced Planck constant to solve energy of light particles called photons. Boltzmann introduced Boltzmann constant to explain the kinetic energy of gas particles. Similarly, consciousness constant Consciere $\mathrm{C}$ is a physical constant of brain waves to solve consciousness. The value of consciousness constant Consciere $\mathrm{C}$ could not be determined since brain energy is too weak to measure with current technology. But as technology advances, it would be possible to measure the brain energy, hence determine the value of Consciere $\mathrm{C}$ by calculating the frequencies and amplitudes of the measured brain waves.

\section{Acknowledgements}

None.

\section{Conflicts of interest}

The author declares that there is no conflict of interest.

\section{References}

1. Das T. Consciousness as a function of brain waves and physical constant Conscire. NeuroQuantology. 2017;15(3).

2. Tapan Das. Brain Waves Create Consciousness. Int J of Dev Res. 2018;8(6):20910-20912.

3. Stanislas Dehaene. Consciousness and the Brain. Penguin Group, New York. 
4. Frank Amthor. Neuroscience. John Wiley\& Sons. USA: New Jersey. 2014.

5. https://inews.co.uk/news/long-reads/i-overwhelming-feeling-love/

6. Borjigin $\mathrm{J}$, Lee Yu, Liu $\mathrm{T}$, et al. Surge of neurophysiological coherence and connectivity in the dying brain. Proc Natl Acad Sci. 2013:110(35):14432-14437.

\section{Appendix}

Fourier transform converts waveform data in the time domain into the frequency domain. The Fourier transform accomplishes this by breaking down the original time-based waveform into a series of sinusoidal terms, each with a unique magnitude, frequency, and phase. This process, in effect, converts a waveform in the time domain that is difficult to describe mathematically into a more manageable series of sinusoidal functions that when added together, exactly reproduce the original waveform.

Let us analyze brain waves of a person in problem solving state. In this case, the dominant waves will be in Beta range, but there will be additional waves in Gamma, Alpha and even Theta ranges. Let us call the brain wave frequencies in Beta range as $f_{b 1}, f_{b 2}, f_{b 3}, f_{b 4}$ and $f_{b 5}$ and their amplitudes $A_{b 1}, A_{b 2}, A_{b 3}, A_{b 4}$ and $A_{b 5}$, in Gamma range $f_{g 1}, f_{g 2}, f_{g 3}$ and their amplitudes $A_{g 1}, A_{g 2}$ and $A_{g 3}$. Similarly, brain waves and brain wave frequency in Alpha range $f_{a 1}$ and its amplitude $A_{a 1}$. Hence the total brain wave energy BWE would be

$$
\begin{aligned}
& B W E=C \cdot A_{b 1}^{2} \cdot f_{b 1}+C \cdot A_{b 2}^{2} \cdot f_{b 2}+C \cdot A_{b 3}^{2} \cdot f_{g 3}+C \cdot A_{b 4}^{2} \cdot f_{g 4}+C \cdot A_{b 5}^{2} \cdot f_{g 5}+C \cdot A_{g 1}^{2} \cdot f_{b 1}+ \\
& C \cdot A_{g 1}^{2} \cdot f_{b 2}+C \cdot A_{g 3}^{2} \cdot f_{b 3}+C \cdot A_{a 1}^{2} \cdot f_{a 1} . \\
= & C\left(A_{b 1}^{2} \cdot f_{b 1}+A_{b 2}^{2} \cdot f_{b 2}+A_{b 3}^{2} \cdot f_{b 3}+A_{b 4}^{2} \cdot f_{b 4}+A_{b 5}^{2} \cdot f_{b 5}+A_{g 1}^{2} \cdot f_{g 1}+A_{g 2}^{2} \cdot f_{g 2}+A_{g 3}^{2} \cdot f_{g 3}+A_{a 1}^{2} \cdot f_{a 1}\right),
\end{aligned}
$$

since energy of a brain wave is proportional to the square of its amplitude.

If we know the value of BWE, then from the values of frequencies and amplitudes obtained by Fourier transforms of the measured waveforms, we can calculate the value of Consciere C. 